KYUNGPOOK Math. J. 56(2016), 57-68

http://dx.doi.org/10.5666/KMJ.2016.56.1.57

pISSN 1225-6951 eISSN 0454-8124

(C) Kyungpook Mathematical Journal

\title{
On Approximation by Matrix Means of the Multiple Fourier Series in the Hölder Metric
}

UG̃UR DEG̃ER

Mersin University, Faculty of Science and Literature, Department of Mathematics, 33343 Mersin - Turkey

e-mail : degpar@hotmail.com and udeger@mersin.edu.tr

ABSTRACT. In this work, we shall give the degree of approximation for functions belonging to Hölder class by matrix summability method of multiple Fourier series in the Hölder metric.

\section{Introduction and some Notations}

Suppose that $f(x, y)$ is integrable in the sense of Lebesgue over the square $S^{2}:=S(-\pi, \pi ;-\pi, \pi)$ and of period $2 \pi$ in $x$ and in $y$. If $f(x, y)$ is defined only on the square $S^{2}$, we extend it periodically onto the whole $x y$-plane. The double Fourier series of $f(x, y)$ can be written in the form

$$
\begin{gathered}
f(x, y) \sim \sum_{m, n \in \mathbb{N}} \lambda_{m n}\left[\eta_{m n} \cos m x \cos n y+\mu_{m n} \sin m x \cos n y\right. \\
\left.+\rho_{m n} \cos m x \sin n y+\zeta_{m n} \sin m x \sin n y\right]
\end{gathered}
$$

where

$$
\lambda_{m n}= \begin{cases}1 / 4, & m=n=0 \\ 1 / 2, & m>0, n=0 \vee m=0, n>0 \\ 1, & m>0, n>0\end{cases}
$$

and the coefficients $\eta_{m n}, \mu_{m n}, \rho_{m n}$ and $\zeta_{m n}$ are calculated by the formulas

Received May 8, 2013; accepted October 7, 2015.

2010 Mathematics Subject Classification: 40B05, 40C05, 40G05, 42A10, 42A24.

Key words and phrases: Trigonometric approximation, Multiple Fourier series, Lipschitz class, Matrix means, Hölder metric. 


$$
\begin{aligned}
& \eta_{m n}=\frac{1}{\pi^{2}} \iint_{S^{2}} f(x, y) \cos m x \cos n y d x d y, \\
& \mu_{m n}=\frac{1}{\pi^{2}} \iint_{S^{2}} f(x, y) \sin m x \cos n y d x d y, \\
& \rho_{m n}=\frac{1}{\pi^{2}} \iint_{S^{2}} f(x, y) \cos m x \sin n y d x d y, \\
& \zeta_{m n}=\frac{1}{\pi^{2}} \iint_{S^{2}} f(x, y) \sin m x \sin n y d x d y,
\end{aligned}
$$

for $m=0,1,2, \ldots$ and $n=0,1,2 \ldots$ Now let

$$
\begin{aligned}
s_{m n}(x, y) & =\sum_{i=0}^{m} \sum_{j=0}^{n}\left[\eta_{i j} \cos i x \cos j y+\mu_{i j} \sin i x \cos j y\right. \\
& \left.+\rho_{i j} \cos i x \sin j y+\zeta_{i j} \sin i x \sin j y\right] .
\end{aligned}
$$

The quantity $s_{m n}(x, y)(m=0,1,2, \ldots ; n=0,1,2 \ldots)$ are called the partial sums of double Fourier series. According to (1.1), we know that

$$
s_{m n}(x, y)=\frac{1}{\pi^{2}} \iint_{S^{2}} f(x+u, y+v) \frac{[\sin (m+1 / 2) u][\sin (n+1 / 2) v]}{4 \sin (u / 2) \sin (v / 2)} d u d v .
$$

Moreover, let

$$
\tau_{m n}(x, y)=\tau_{m n}(f ; A, U ; x, y):=\sum_{i=0}^{m} \sum_{j=0}^{n} a_{m i} b_{n j} s_{i j}(x, y), \quad \forall m, n \geq 0
$$

where $A \equiv\left(a_{m, i}\right)$ and $U \equiv\left(b_{n, j}\right)$ are lower triangular infinite matrices such that:

$$
a_{m, i}=\left\{\begin{array}{ll}
\geq 0, & i \leq m ; \\
0, & i>m
\end{array} \quad(i, m=0,1,2, \ldots) \quad \wedge \quad \sum_{i=0}^{m} a_{m, i}=1\right.
$$

and

$$
b_{n, j}=\left\{\begin{array}{ll}
\geq 0, & j \leq n ; \\
0, & j>n
\end{array} \quad(j, n=0,1,2, \ldots) \quad \wedge \quad \sum_{j=0}^{n} a_{n, j}=1 .\right.
$$

The double Fourier series of the function $f(x, y)$ is called to be $(A, U)$-summable to a finite number $s$, if $\tau_{m n}(x, y) \rightarrow s$ as $m, n \rightarrow \infty$. The condition of regularity for 
double matrix summability means are given by

$$
\begin{aligned}
& \sum_{i=0}^{m} \sum_{j=0}^{n} a_{m i} b_{n j} \rightarrow 1, \text { as } m, n \rightarrow \infty, \\
& \lim _{m, n} \sum_{j=0}^{n}\left|a_{m i} b_{n j}\right|=0, \text { for each } i=1,2, \ldots, \\
& \lim _{m, n} \sum_{i=0}^{m}\left|a_{m i} b_{n j}\right|=0, \text { for each } \quad j=1,2, \ldots
\end{aligned}
$$

Let

$$
H_{\alpha}=\left\{f \in C_{2 \pi}:|f(x)-f(y)| \leq K|x-y|^{\alpha}\right\}
$$

where $K$ is a positive constant, not necessarily the same at each occurrence. It is known that $H_{\alpha}$ is a Banach space(see Prösdorff, [7]) with the norm $\|\cdot\|_{\alpha}$ defined by

$$
\|f\|_{\alpha}=\|f\|_{C}+\sup _{x \neq y} \Delta^{\alpha} f(x, y)
$$

where

$$
\Delta^{\alpha} f(x, y)=\frac{|f(x)-f(y)|}{|x-y|^{\alpha}}(x \neq y),
$$

by convention $\Delta^{0} f(x, y)=0$ and

$$
\|f\|_{C}=\sup _{x \in[-\pi, \pi]}|f(x)| .
$$

The metric induced by the norm (1.5) on $H_{\alpha}$ is called the Hölder metric. Prösdorff has been studied the degree of approximation in the Hölder metric and proved the following theorem:

Theorem A.([7]) Let $f \in H_{\alpha}(0<\alpha \leq 1)$ and $0 \leq \beta<\alpha \leq 1$. Then

$$
\left\|\sigma_{n}(f)-f\right\|_{\beta}=O(1) \begin{cases}n^{\beta-\alpha} & , 0<\alpha<1 \\ n^{\beta-1} \ln n & , \alpha=1\end{cases}
$$

where $\sigma_{n}(f)$ is Fejér means of the Fourier series of $f$.

The case $\beta=0$ in Theorem $\mathrm{A}$ is owing to Alexits [1]. Chandra obtained a generalization of Theorem A in the Woronoi-Nörlund transform [2]. In [6], Mohapatra and Chandra considered the problem by matrix means of the Fourier series of $f \in H_{\alpha}$. In the one-dimensional case, these problems have been studied in detail. Naturally, similar problems are considered for the periodic functions with two variables. Stepanets investigated the problem of the approximation of functions $f(x, y)$, $2 \pi$-periodic with respect to each of the variables by the partial sums of their Fourier sums and under the some conditions in $[9,10]$. In [5], Lal studied the approximation 
of functions belonging to Lipschitz class by matrix summability method for double Fourier series under the uniform norm.

The Hölder class for $f(x, y)$ continuous functions periodic in both variables with period $2 \pi$ is defined as

$$
H_{(\alpha, \beta)}=\left\{f:|f(x, y ; z, w)|:=|f(x, y)-f(z, w)| \leq C_{1}\left(|x-z|^{\alpha}+|y-w|^{\beta}\right)\right\}
$$

for some $\alpha, \beta>0$ and for all $x, y, z, w$ where $C_{1}$ is a positive constant may depend on $f$, but not on $x, y, z, w$. This class of functions is also called Lipschitz class and denoted by $\operatorname{Lip}(\alpha, \beta)$. It can be easily verified that $H_{\alpha, \beta}$ is a Banach space with the norm $\|\cdot\|_{\alpha, \beta}$ defined by

$$
\|f\|_{\alpha, \beta}=\|f\|_{C}+\sup _{x \neq z, y \neq w} \Delta^{\alpha, \beta} f(x, y ; z, w)
$$

where

$$
\Delta^{\alpha, \beta} f(x, y ; z, w)=\frac{|f(x, y)-f(z, w)|}{|x-z|^{\alpha}+|y-w|^{\beta}}(x \neq z, y \neq w)
$$

by convention $\Delta^{0,0} f(x, y ; z, w)=0$ and

$$
\|f\|_{C}=\sup _{(x, y) \in S^{2}}|f(x, y)| .
$$

Moreover, a function $f$ in $\operatorname{Lip}(\alpha, \beta)$ is said to belong to the little Lipschitz class $\operatorname{lip}(\alpha, \beta)$ if

$$
\lim _{z \rightarrow x, w \rightarrow y}\left(|x-z|^{\alpha}+|y-w|^{\beta}\right)^{-1}|f(x, y ; z, w)|=0
$$

uniformly in $(x, y)$. The aim of this paper is as follows. First, the approximation to functions $f(x, y)$ belonging to these Lipschitz classes is given by matrix summability method of double Fourier series in accordance with the norm in (1.7). Later the approximation is generalized to the $N$-multiple Fourier series.

Throughout this paper, we shall also use the following notations:

$$
\begin{aligned}
\Psi(u, v) & :=\Psi(x, y ; u, v):=\frac{1}{4}\{f(x+u, y+v)+f(x+u, y-v) \\
& +f(x-u, y+v)+f(x-u, y-v)-4 f(x, y)\}
\end{aligned}
$$

and

$$
F(u, v)=\Phi(u, v)-\Psi(u, v)
$$

where $\Phi(u, v):=\Psi(z, w ; u, v)$. Since $f(x, y) \in H_{(\alpha, \beta)}$, it is clear that

$$
|F(u, v)|=O\left(|x-z|^{\alpha}+|y-w|^{\beta}\right) .
$$




\section{In Case of Double Fourier Series}

The approximation by matrix means for double Fourier series is as follows with respect to Hölder metric.

Theorem 2.1. Assume $A \equiv\left(a_{m, i}\right)$ and $U \equiv\left(b_{n, j}\right)$ are lower triangular matrices where $\left(a_{m, i}\right)$ and $\left(b_{n, j}\right)$ are nondecreasing sequences with respect to $i \leq m$ and $j \leq n$ satisfying the conditions (1.2) and (1.3), respectively such that double matrix method $(A, U)$ is regular. If $f(x, y)$ is a function of period $2 \pi$ in $x$ and $y$ Lebesgue integrable in $S^{2}$ belonging to the class $H_{(\alpha, \beta)}$ for $0<\alpha, \beta \leq 1$, then

$\left\|\tau_{m n}-f\right\|_{\alpha, \beta}=O(1) \begin{cases}(m+1)^{-\alpha}+(n+1)^{-\beta} & , 0<\alpha<1,0<\beta<1 ; \\ \frac{\log ((m+1) \pi)}{(m+1)}+\frac{\log ((n+1) \pi)}{(n+1)} & , \alpha=\beta=1\end{cases}$

for $m, n=0,1,2, \ldots$

For small Lipschitz class, the analogy of the Theorem can be written if " $O$ " is replaced by " $o$ " as $m, n \rightarrow \infty$ independently one another, and $f \in \operatorname{Lip}(\alpha, \beta)$ is replaced by $f \in \operatorname{lip}(\alpha, \beta)$ for $0<\alpha, \beta<1$. We don't enter in details.

Furthermore, double matrix summability method gives us the following means for some important cases:

- $(C, 1,1)$ means, when $a_{m, i}=\frac{1}{m+1}$ and $b_{n, j}=\frac{1}{n+1}$ for all $i$ and $j$, respectively [3];

- $\left(N, p_{m}, q_{n}\right)$ means, when $a_{m, i}=\frac{p_{m-i}}{P_{m}}$ and $b_{n, j}=\frac{q_{n-j}}{Q_{n}}$; where $P_{m}=\sum_{k=0}^{m} p_{k} \neq$ 0 and $Q_{n}=\sum_{k=0}^{n} q_{k} \neq 0[4]$;

- $(H, 1,1)$ means, when $a_{m, i}=\frac{1}{(m-i+1) \log m}$ and $b_{n, j}=\frac{1}{(n-j+1) \log n}$ [8].

Taking into account the first two case above, we write the following results.

Corollary 2.2. If $f(x, y)$ is a function of period $2 \pi$ in $x$ and $y$ Lebesgue integrable in $S^{2}$ belonging to the class $H_{(\alpha, \beta)}$ for $0<\alpha, \beta \leq 1$, then

$\left\|\sigma_{m n}-f\right\|_{\alpha, \beta}=O(1) \begin{cases}(m+1)^{-\alpha}+(n+1)^{-\beta} & , 0<\alpha<1,0<\beta<1 ; \\ \frac{\log ((m+1) \pi)}{(m+1)}+\frac{\log ((n+1) \pi)}{(n+1)} & , \alpha=\beta=1\end{cases}$

for $m, n=0,1,2, \ldots$, where

$$
\sigma_{m n}(x, y)=\frac{1}{(m+1)(n+1)} \sum_{i=0}^{m} \sum_{j=0}^{n} s_{i j}(x, y), \quad \forall m, n \geq 0 .
$$


Corollary 2.3. If $f(x, y)$ is a function of period $2 \pi$ in $x$ and $y$ Lebesgue integrable in $S^{2}$ belonging to the class $H_{(\alpha, \beta)}$ for $0<\alpha, \beta<1$, then

$$
\left\|\mathbf{N}_{m n}-f\right\|_{\alpha, \beta}=O(1)\left\{(m+1)^{-\alpha}+(n+1)^{-\beta}\right\}
$$

for $m, n=0,1,2, \ldots$, where

$$
\mathbf{N}_{m n}(x, y)=\frac{1}{P_{m} Q_{n}} \sum_{i=0}^{m} \sum_{j=0}^{n} p_{m-i} q_{n-j} s_{i j}(x, y), \quad \forall m, n \geq 0 .
$$

Before giving the proof of Theorem 2.1, we need the following auxiliary results.

Lemma 2.4. Let $\left(a_{m, i}\right)$ and $\left(b_{n j}\right)$ be real nonnegative and nondecreasing sequence with (1.2) and (1.3), respectively.

(i) For $0<u \leq 1 /(m+1)$, we have $K_{m}(u)=O(m+1)$ where

$$
K_{m}(u):=\frac{1}{\pi} \sum_{i=0}^{m} a_{m, i} \frac{\sin \left(i+\frac{1}{2}\right) u}{\sin \left(\frac{u}{2}\right)} .
$$

(ii) For $0<v \leq 1 /(n+1)$, we have $K_{n}(v)=O(n+1)$ where

$$
K_{n}(v):=\frac{1}{\pi} \sum_{j=0}^{n} b_{n, j} \frac{\sin \left(j+\frac{1}{2}\right) v}{\sin \left(\frac{v}{2}\right)} .
$$

This is easily proved by an elementary calculation.

Lemma 2.5. ([5]) Assume that $\left(a_{m, i}\right)$ and $\left(b_{n j}\right)$ be real nonnegative and nondecreasing sequence with $i \leq m$ and $j \leq n$, respectively.

(i) For $1 /(n+1)<v \leq \pi$ and any $n \in \mathbb{N}$, we have

$$
K_{n}(v)=O\left(\frac{B_{n, \sigma}}{v}\right)
$$

where $B_{n, \sigma}=\sum_{j=n-\sigma}^{n} b_{n j}$ and $\sigma$ denote integer part of $\frac{1}{v}$.

(ii) For $1 /(m+1)<u \leq \pi$ and any $m \in \mathbb{N}$, we have

$$
K_{m}(u)=O\left(\frac{A_{m, \kappa}}{u}\right)
$$

where $A_{m, \kappa}=\sum_{i=m-\kappa}^{m} a_{m i}$ and $\kappa$ denote integer part of $\frac{1}{u}$. 


\section{Proof of the Theorem 2.1}

Proof. We know that

$$
s_{i j}(x, y)-f(x, y)=\frac{1}{\pi^{2}} \int_{0}^{\pi} \int_{0}^{\pi} \Psi(u, v) \frac{[\sin (i+1 / 2) u][\sin (j+1 / 2) v]}{\sin (u / 2) \sin (v / 2)} d u d v .
$$

Taking into account (3.1) and $\tau_{m n}(x, y)$ that double matrix means of $s_{m n}(x, y)$, we write

$$
\begin{gathered}
\tau_{m n}(x, y)-f(x, y)=\sum_{i=0}^{m} \sum_{j=0}^{n} a_{m i} b_{n j}\left\{s_{i j}(x, y)-f(x, y)\right\} \\
=\frac{1}{\pi^{2}} \int_{0}^{\pi} \int_{0}^{\pi} \Psi(u, v) \sum_{i=0}^{m} \sum_{j=0}^{n} a_{m i} b_{n j} \frac{[\sin (i+1 / 2) u][\sin (j+1 / 2) v]}{\sin (u / 2) \sin (v / 2)} d u d v \\
=\int_{0}^{\pi} \int_{0}^{\pi} \Psi(u, v) K_{m}(u) K_{n}(v) d u d v
\end{gathered}
$$

Let us estimate that

$$
\sup _{x \neq z, y \neq w} \frac{\left|\tau_{m n}(x, y)-f(x, y)-\left(\tau_{m n}(z, w)-f(z, w)\right)\right|}{|x-z|^{\alpha}+|y-w|^{\beta}}=O(1) .
$$

$\left|\tau_{m n}(x, y)-f(x, y)-\left(\tau_{m n}(z, w)-f(z, w)\right)\right|=\left|\int_{0}^{\pi} \int_{0}^{\pi} F(u, v) K_{m}(u) K_{n}(v) d u d v\right|$

$\leq\left(\int_{0}^{\frac{1}{(m+1)}} \int_{0}^{\frac{1}{(n+1)}}+\int_{0}^{\frac{1}{(m+1)}} \int_{\frac{1}{(n+1)}}^{\pi}+\int_{\frac{1}{(m+1)}}^{\pi} \int_{0}^{\frac{1}{(n+1)}}+\int_{\frac{1}{(m+1)}}^{\pi} \int_{\frac{1}{(n+1)}}^{\pi}\right)\left|F(u, v) K_{m}(u) K_{n}(v)\right| d u d v$

$$
=: J_{1}+J_{2}+J_{3}+J_{4} .
$$

Therefore, from (1.8) and Lemma 2.4, we obtain

$$
\begin{aligned}
J_{1} & =\int_{0}^{1 /(m+1)} \int_{0}^{1 /(n+1)}\left|F(u, v) K_{m}(u) K_{n}(v)\right| d u d v \\
(3.4) & =(m+1)(n+1) \int_{0}^{1 /(m+1)} \int_{0}^{1 /(n+1)}|F(u, v)| d u d v=O\left(|x-z|^{\alpha}+|y-w|^{\beta}\right)
\end{aligned}
$$


for $0<\alpha, \beta \leq 1$. By using Lemma 2.4, Lemma 2.5 and again (1.8), then we have

$$
\begin{aligned}
J_{2} & =\int_{0}^{1 /(m+1)} \int_{1 /(n+1)}^{\pi}\left|F(u, v) K_{m}(u) K_{n}(v)\right| d u d v \\
& =(m+1) \int_{0}^{1 /(m+1)} \int_{1 /(n+1)}^{\pi}|F(u, v)| \frac{B_{n, \sigma}}{v} d u d v=O\left(|x-z|^{\alpha}+|y-w|^{\beta}\right) \int_{1 /(n+1)}^{\pi} \frac{B_{n, \sigma}}{v} d v \\
& \leq O\left(|x-z|^{\alpha}+|y-w|^{\beta}\right) \int_{1 /(n+1)}^{\pi} \frac{B_{n, 1 / v}}{v} d v \\
(3.5) & =O\left(|x-z|^{\alpha}+|y-w|^{\beta}\right) \int_{1 / \pi}^{(n+1)} \frac{B_{n, t}}{t} d t=O\left(|x-z|^{\alpha}+|y-w|^{\beta}\right)
\end{aligned}
$$

since $\frac{B_{n, t}}{t}$ is monotonic increasing. Similarly, we can prove that

$$
J_{3}=\int_{1 /(m+1)}^{\pi} \int_{0}^{1 /(n+1)}\left|F(u, v) K_{m}(u) K_{n}(v)\right| d u d v=O\left(|x-z|^{\alpha}+|y-w|^{\beta}\right)
$$

and

$$
J_{4}=\int_{1 /(m+1)}^{\pi} \int_{1 /(n+1)}^{\pi}\left|F(u, v) K_{m}(u) K_{n}(v)\right| d u d v=O\left(|x-z|^{\alpha}+|y-w|^{\beta}\right) .
$$

By combining (3.3)-(3.7), we obtain (3.2). On the other hand, we know that from [5]

(3.8) $\left\|\tau_{m n}-f\right\|_{C}=O(1) \begin{cases}(m+1)^{-\alpha}+(n+1)^{-\beta} & , 0<\alpha, \beta<1 ; \\ \frac{\log ((m+1) \pi e)}{(m+1)}+\frac{\log ((n+1) \pi e)}{(n+1)} & , \alpha=\beta=1\end{cases}$

for $m, n=0,1,2, \ldots$ Since $\log e<\log (m+1) \pi$ and $\log e<\log (n+1) \pi$, we omit the number " $e "$ in the formula (3.8). Therefore, according to (3.2) and (3.8), the proof of Theorem 2.1 is completed.

4. In Case of $N$-Multiple Fourier Series, $N \geq 3$.

Let $f\left(x_{1}, \ldots, x_{N}\right)$ is integrable over the $N$ dimensional cube $S^{N}$ and of period 
$2 \pi$ in each variable. The $N$-multiple Fourier series of $f\left(x_{1}, \ldots, x_{N}\right)$ can be written in the form

$$
f\left(x_{1}, \ldots, x_{N}\right) \sim \sum_{m_{1} \in \mathbb{Z}} \sum_{m_{2} \in \mathbb{Z}} \cdots \sum_{m_{N} \in \mathbb{Z}} c_{m_{1}, m_{2}, \ldots, m_{N}} e^{i\left(m_{1} x_{1}+m_{2} x_{2}+\cdots+m_{N} x_{N}\right)} .
$$

where $c_{m_{1}, m_{2}, \ldots, m_{N}}$ is the Fourier coefficients of $f$ (see, [11, p. 300]). The series is denoted by $S[f]$ and the partial sums of it are given by

$$
S_{m_{1} m_{2} \ldots m_{N}}\left(x_{1}, \ldots, x_{N}\right):=\pi^{-N} \int_{-\pi}^{\pi} \ldots \int_{-\pi}^{\pi} f\left(x_{1}+t_{1}, \ldots, x_{N}+t_{N}\right) \prod_{j=1}^{N} D_{m_{j}}\left(t_{j}\right) d t_{1} \ldots d t_{N}
$$

where $D_{m_{j}}\left(t_{j}\right)$ are the Dirichlet kernels for each $j$. Moreover, similar to the twodimensional, we can write

$$
\begin{gathered}
\tau_{m_{1} m_{2} \cdots m_{N}}\left(x_{1}, \ldots, x_{N}\right)=: \tau_{m_{1} m_{2} \cdots m_{N}}\left(f ;\left\{A_{k}\right\}_{1}^{N} ; x_{1}, \ldots, x_{N}\right) \\
:=\sum_{i_{1}=0}^{m_{1}} \sum_{i_{2}=0}^{m_{2}} \ldots \sum_{i_{N}=0}^{m_{N}} a_{m_{1} i_{1}} \ldots a_{m_{N} i_{N}} S_{i_{1} i_{2} \cdots i_{N}}\left(x_{1}, \ldots, x_{N}\right)
\end{gathered}
$$

for all $m_{k} \geq 0$. Here $\left\{A_{k}\right\}_{k=1}^{N} \equiv\left\{\left(a_{m_{k}, i_{k}}\right)\right\}_{k=1}^{N}$ are lower triangular infinite matrices such that:

$$
a_{m_{k}, i_{k}}=\left\{\begin{array}{ll}
\geq 0, & i_{k} \leq m_{k} ; \\
0, & i_{k}>m_{k}
\end{array} \quad\left(m_{k}, i_{k}=0,1,2, \ldots\right) \quad \wedge \quad \sum_{i_{k}=0}^{m_{k}} a_{m_{k}, i_{k}}=1\right.
$$

for each $k=1,2, \ldots, N$. The $N$-multiple Fourier series of function $f\left(x_{1}, \ldots, x_{N}\right)$ is called to be $\left(A_{1}, \ldots, A_{N}\right)$-summable to a finite number $\ell$, if $\tau_{m_{1} m_{2} \cdots m_{N}}\left(x_{1}, \ldots, x_{N}\right)$ $\rightarrow \ell$ as $m_{1}, m_{2}, \cdots, m_{N} \rightarrow \infty$. The condition of regularity for $N$-multiple matrix summability means are given by

$$
\begin{gathered}
\sum_{i_{1}=0}^{m_{1}} \sum_{i_{2}=0}^{m_{2}} \ldots \sum_{i_{N}=0}^{m_{N}}\left(a_{m_{1} i_{1}} \ldots a_{m_{N} i_{N}}\right) \rightarrow 1, \text { as } \quad m_{1}, m_{2}, \ldots, m_{N} \rightarrow \infty, \\
\lim _{m_{i}} \sum_{i_{2}=0}^{m_{2}} \sum_{i_{3}=0}^{m_{3}} \ldots \sum_{i_{N}=0}^{m_{N}}\left(a_{m_{1} i_{1}} \ldots a_{m_{N} i_{N}}\right)=0, \text { for each } i_{1}=1,2, \ldots, \\
\lim _{m_{i}} \sum_{i_{1}=0}^{m_{1}} \sum_{i_{3}=0}^{m_{3}} \ldots \sum_{i_{N}=0}^{m_{N}}\left(a_{m_{1} i_{1}} \ldots a_{m_{N} i_{N}}\right)=0, \text { for each } i_{2}=1,2, \ldots, \\
\vdots \\
\lim _{m_{i}} \sum_{i_{1}=0}^{m_{1}} \sum_{i_{2}=0}^{m_{2}} \ldots \sum_{i_{N-1}=0}^{m_{N-1}}\left(a_{m_{1} i_{1}} \ldots a_{m_{N} i_{N}}\right)=0, \text { for each } i_{N}=1,2, \ldots
\end{gathered}
$$


Next, we give the notion of Lipschitz classes of functions on $S^{N}$. Let $f\left(x_{1}, \ldots, x_{N}\right)$ be a continuous periodic function with period $2 \pi$ in each variable. The function $f$ belongs to the Lipschitz class $\operatorname{Lip}\left(\alpha_{1}, \alpha_{2}, \ldots, \alpha_{N}\right)\left(\right.$ or $\left.H_{\left(\alpha_{1}, \alpha_{2}, \ldots, \alpha_{N}\right)}\right)$ for some $\alpha_{1}, \alpha_{2}, \ldots, \alpha_{N} \geq 0$ if there exists a constant $K_{1}$ such that

$$
\left|f\left(x_{1}, \ldots, x_{N} ; y_{1}, \ldots, y_{N}\right)\right|:=\left|f\left(x_{1}, \ldots, x_{N}\right)-f\left(y_{1}, \ldots, y_{N}\right)\right| \leq K_{1} \sum_{k=1}^{N}\left|x_{k}-y_{k}\right|^{\alpha_{k}}
$$

for all $x_{k}, y_{k}$ where $k=1, \ldots, N$. Furthermore, a function $f$ in $\operatorname{Lip}\left(\alpha_{1}, \alpha_{2}, \ldots, \alpha_{N}\right)$ is said to belong to little Lipschitz class $\operatorname{lip}\left(\alpha_{1}, \alpha_{2}, \ldots, \alpha_{N}\right)$ if

$$
\lim _{y_{1} \rightarrow x_{1}, \cdots, y_{N} \rightarrow x_{N}} \frac{\left|f\left(x_{1}, \ldots, x_{N} ; y_{1}, \ldots, y_{N}\right)\right|}{\sum_{k=1}^{N}\left|x_{k}-y_{k}\right|^{\alpha_{k}}}=0
$$

uniformly in $\left(x_{1}, \ldots, x_{N}\right)$.

The function space $H_{\left(\alpha_{1}, \alpha_{2}, \ldots, \alpha_{N}\right)}$ is a Banach space with respect to the norm $\|\cdot\|_{\alpha_{1}, \alpha_{2}, \ldots, \alpha_{N}}$ defined by

$$
\|f\|_{\alpha_{1}, \alpha_{2}, \ldots, \alpha_{N}}=\|f\|_{C}+\sup _{x_{1} \neq y_{1}, \ldots, x_{N} \neq y_{N}} \Delta^{\alpha_{1}, \alpha_{2}, \ldots, \alpha_{N}} f\left(x_{1}, \ldots, x_{N} ; y_{1}, \ldots, y_{N}\right)
$$

where

$$
\Delta^{\alpha_{1}, \alpha_{2}, \ldots, \alpha_{N}} f\left(x_{1}, \ldots, x_{N} ; y_{1}, \ldots, y_{N}\right)=\frac{\left|f\left(x_{1}, \ldots, x_{N} ; y_{1}, \ldots, y_{N}\right)\right|}{\sum_{k=1}^{N}\left|x_{k}-y_{k}\right|^{\alpha_{k}}}
$$

for $x_{1} \neq y_{1}, \ldots, x_{N} \neq y_{N}$ by convention $\Delta^{0, \ldots, 0} f\left(x_{1}, \ldots, x_{N} ; y_{1}, \ldots, y_{N}\right)=0$ and

$$
\|f\|_{C}=\sup _{\left(x_{1}, \ldots, x_{N}\right) \in S^{N}}\left|f\left(x_{1}, \ldots, x_{N}\right)\right| .
$$

Now as an extension of Theorem 2.1, we write the following theorem.

Theorem 4.1. Let $\left\{A_{k}\right\}_{k=1}^{N} \equiv\left\{\left(a_{m_{k}, i_{k}}\right)\right\}_{k=1}^{N}, N \geq 3$, are lower triangular matrices where $\left\{\left(a_{m_{k}, i_{k}}\right)\right\}_{k=1}^{N}$ are nondecreasing sequences with respect to $i_{k} \leq m_{k}$, $k=1, \ldots, N$, satisfying the conditions (4.1), respectively such that $N$-multiple matrix method $\left(A_{1}, A_{2}, \ldots, A_{N}\right)$ is regular. If $f\left(x_{1}, x_{2}, \ldots, x_{N}\right)$ is a function of period $2 \pi$ in each variable Lebesgue integrable in $S^{N}$ belonging to the class $H_{\left(\alpha_{1}, \alpha_{2}, \ldots, \alpha_{N}\right)}$ for $0<\alpha_{1}, \alpha_{2}, \ldots, \alpha_{N} \leq 1$, then

$$
\left\|\tau_{m_{1} m_{2} \cdots m_{N}}-f\right\|_{\alpha_{1}, \alpha_{2}, \ldots, \alpha_{N}}=O(1) \begin{cases}\sum_{k=1}^{N}\left(m_{k}+1\right)^{-\alpha_{k}} & , 0<\alpha_{1}, \alpha_{2}, \ldots, \alpha_{N}<1 ; \\ \sum_{k=1}^{N} \frac{\log \left(\left(m_{k}+1\right) \pi\right)}{\left(m_{k}+1\right)} & , \alpha_{1}=\alpha_{2}=\cdots=\alpha_{N}=1,\end{cases}
$$


for $m_{k}=0,1,2, \ldots$, where $k=1,2, \ldots, N$ and $N \geq 3$ is a fixed integer.

Proof. One needs the extensions of Lemma 2.4 and Lemma 2.5 with respect to each variable from double to $N$-multiple. After this, the proof runs along the same lines as that of Theorem 2.1.

Let $0<\alpha_{1}, \alpha_{2}, \ldots, \alpha_{N}<1$. The analogy of statement in the Theorem 2.1 can be written if " $O$ " is replaced by " $O$ " as $m_{1}, m_{2}, \cdots, m_{N} \rightarrow \infty$, and $f \in H_{\left(\alpha_{1}, \alpha_{2}, \ldots, \alpha_{N}\right)}$ is replaced by $f \in \operatorname{lip}\left(\alpha_{1}, \alpha_{2}, \ldots, \alpha_{N}\right)$.

$N$ - multiple matrix summability method gives us the $(C, 1,1, \ldots, 1)$ means, when $a_{m_{k}, i_{k}}=\frac{1}{m_{k}+1}$ for all $i_{k},(k=1,2, \ldots, N)$ [11]. Then, it will be in the form

$$
\sigma_{m_{1} m_{2} \cdots m_{N}}\left(x_{1}, \ldots, x_{N}\right)=\left(\prod_{k=1}^{N} \frac{1}{m_{k}+1}\right) \sum_{i_{1}=0}^{m_{1}} \sum_{i_{2}=0}^{m_{2}} \ldots \sum_{i_{N}=0}^{m_{N}} S_{i_{1} i_{2} \cdots i_{N}}\left(x_{1}, \ldots, x_{N}\right)
$$

Therefore, we observe the next result from the Theorem 4.1.

Corollary 4.2. If $f\left(x_{1}, x_{2}, \ldots, x_{N}\right)$ is a function of period $2 \pi$ in each variable Lebesgue integrable in $S^{N}$ belonging to the class $H_{\left(\alpha_{1}, \alpha_{2}, \ldots, \alpha_{N}\right)}$ for $0<$ $\alpha_{1}, \alpha_{2}, \ldots, \alpha_{N} \leq 1$, then

$\left\|\sigma_{m_{1} m_{2} \cdots m_{N}}-f\right\|_{\alpha_{1}, \alpha_{2}, \ldots, \alpha_{N}}=O(1) \begin{cases}\sum_{k=1}^{N}\left(m_{k}+1\right)^{-\alpha_{k}} & , 0<\alpha_{1}, \alpha_{2}, \ldots, \alpha_{N}<1 ; \\ \sum_{k=1}^{N} \frac{\log \left(\left(m_{k}+1\right) \pi\right)}{\left(m_{k}+1\right)} & , \alpha_{1}=\alpha_{2}=\cdots=\alpha_{N}=1\end{cases}$

for $m_{k}=0,1,2, \ldots$, where $k=1,2, \ldots, N$ and $N \geq 3$ is a fixed integer.

\section{References}

[1] G. Alexits, Convergence problems of orthogonal series, New York: Pergamon Press, (1961).

[2] P. Chandra, On the generalized Fejer means in the metric of the Hölder space, Math. Nachr., 109(1982), 39-45.

[3] Y. S. Chow, On the Cesáro summability of double Fourier series, Tohoku Math. J., 5(1935), 277-283.

[4] E. Hille and J. D. Tamarkin, On the summability of Fourier series, Trans. Amer. Math. Soc., 34(1932), 757-783.

[5] S. Lal, On the approximation of function $f(x, y)$ belonging to Lipschitz class by matrix summability method of double Fourier series, Journal of the Indian Math. Soc., 78(14)(2011), 93-101. 
[6] R. N. Mohapatra and P. Chandra, Degree of approximation of functions in the Hölder metric, Acta Math. Hung., 41 (1-2)(1983), 67-76.

[7] S. Prösdorff, Zur Konvergenz der Fourier reihen Hölder Stetiger Funktionen, Math. Nachr., 69(1975), 7-14.

[8] P. L. Sharma, On the harmonic summability of double Fourier series, Proc. Amer. Math. Soc., 91(1958), 979-986.

[9] A. I. Stepanets, The approximation of certain classes of differentiable periodic functions of two variables by Fourier sums, Ukrainian Mathematical Journal (Translated from Ukrainskii Matematieheskii Zhurnal, 25(5)(1973), 599-609), 26(1973), 498-506.

[10] A. I. Stepanets, Approximation of certain classes of periodic functions of two variables by linear methods of summation of their Fourier series, Ukrainian Mathematical Journal (Translated from Ukrainskii Matematieheskii Zhurnal, 26(2)(1974), 205-215), 26(1974), 168-179.

[11] A. Zygmund, Trigonometric Series, Vol. II, Cambridge University Press, Cambridge, (1959). 